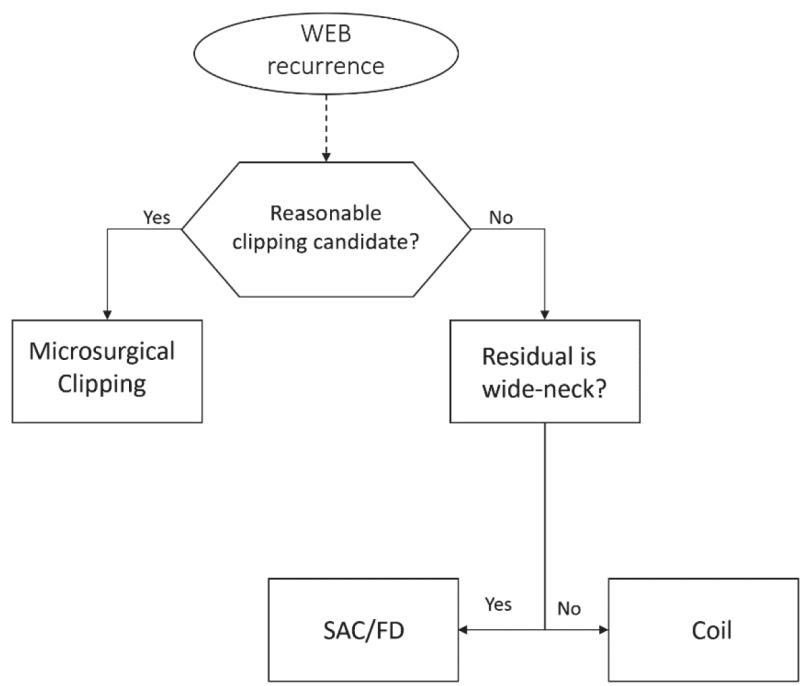

Abstract LB-009 Figure 3

A. Patel: None. N. Alotaibi: None. E. Levy: None. M. Waqas: None. J. Cherian: None. J. Johnson: None. P. Jabbour: None. A. Sweid: None. B. Gross: None. R. Starke: None. A. Puri: None. F. Massari: None. C. Griessenauer: None. J. Catapano: None. C. Rutledge: None. O. Tanweer: None. P. Yashar: None. G. Cortez: None. R. Hanel: None. A. Ducruet: None. F. Albuquerque: None. M. Lawton: None. P. Kan: None.

\section{LB-010 FIVE-YEAR RESULTS OF THE SCENT TRIAL WITH SURPASS FLOW DIVERTERS TO TREAT LARGE OR GIANT WIDE-NECK ANEURYSMS}

${ }^{1} \mathrm{P}$ Meyers ${ }^{*},{ }^{2} \mathrm{~A}$ Wakhloo, ${ }^{3} \mathrm{~A}$ Coon, ${ }^{4} \mathrm{P}$ Kan, ${ }^{5} \mathrm{R}$ Hanel. ${ }^{1}$ Departments of Radiology and Neurological Surgery, Columbia University, New York, NY; ${ }^{2}$ Department of Neurolnterventional Radiology, Beth Israel Lahey Health, Tufts University Medical School, Burlington, MA; ${ }^{3}$ Carondelet Neurosurgical Institute of St. Joseph's Hospital, Tuscon, AZ; ${ }^{4}$ Department of Neurosurgery, University of Texas Medical Center, Galveston, TX; ${ }^{5}$ Lyerly Neurosurgery, Jacksonville, FL

\subsection{6/neurintsurg-2021-SNIS.251}

Introduction The Surpass Flow Diverter provides a minimally invasive, single flow diverter treatment option for patients suffering from large or giant intracranial aneurysms (IA) arising from the internal carotid artery (ICA) to the ICA terminus. Long-term clinical outcomes following the Surpass Flow
Diverter treatment of large or giant wide-neck aneurysms has not been demonstrated.

Objective To report on 5-year safety and efficacy outcomes of patients treated in the Surpass Intracranial Aneurysm Embolization System Trial to treat large or giant wide-neck aneurysms (SCENT Trial).

Methods In this multi-center and prospective trial, 180 aneurysms in 180 subjects were treated with the flow diverter system. Patients were followed per a standardized protocol at 1 month, 6 months and every year through 5 years. Aneurysm occlusion, in-stent stenosis, modified Rankin Scale scores, and complications were recorded.

Results From October 2012 through November 2015, 180 subjects were treated at 26 sites. Five-year clinical follow up completion rate was $96.7 \%$. The primary endpoint of complete aneurysm occlusion without clinically significant parent artery stenosis or retreatment at one year (62.8\%) was previous reported. Aneurysm occlusion (Raymond Class I) for those patients with angiographic follow-up progressively increased over time to $77.8 \%(91 / 117)$ and $90.2 \%(74 / 82)$ at 3 and 5 years. The cumulative proportion of subjects experiencing new or worsening major ipsilateral stroke, as adjudicated by the CEC, was $12.2 \%(22 / 180)$ and $12.8 \%(23 / 180)$ subjects at 3 and 5 years. Four patients $(2.2 \%, 4 / 180)$ experienced aneurysm rupture within the first-week post target aneurysm treatment. No additional late aneurysm ruptures occurred through the five-year follow-up. Five aneurysms $(2.8 \%)$ were retreated in the study. New serious adverse events at 3 and 5 years were noted in 15.4\% (23/149) and $9.8 \%(12 / 123)$ of subjects. Five neurological deaths occurred within one year, and one additional neurological death was reported after the subject's 5-year follow up.

Conclusions Our 5-year findings demonstrate the long-term safety and effectiveness of the Surpass Flow Diverter for intracranial aneurysm treatment, supported by high occlusion rates and low rates of delayed adverse events in this study.

Disclosures P. Meyers: 2; C; Stryker, Medtronic, Penumbra. A. Wakhloo: 1; C; Philips Medical. 2; C; Stryker. 3; C; SCENT trial (Surpass Intracranial Aneurysm Embolization System Pivotal Trial to Treat Large or Giant Wide Neck Aneurysms) presentations. 4; C; InNeuroCo., NovaSignal, Rist, Analytics 4 Life, ThrombX. A. Coon: 2; C; Stryker, Medtronic, Microvention, InNeuroCo. P. Kan: 2; C; Stryker, Imperative Care. 4; C; InNeuroCo. R. Hanel: 1; C; Medtronic, Stryker, Microvention, Cerenovus. 2; C; Stryker, Medtronic, Cerenovus, Microvention. 4; C; Neurvana, Elum, EndoStream, Three Rivers Medical Inc., Rist, Cerebrotech, InNeuroCo.. 6; C; MIVI, Elum, Three Rivers Medical Inc., Shape Medical. 